\title{
HÖRMANDER'S CARLESON THEOREM FOR THE BALL
}

\author{
by S. C. POWER
}

(Received 5 July, 1983)

Let $B=\left\{\left(z_{1}, z_{2}\right) \in \mathbb{C}^{2}:\left|z_{1}\right|^{2}+\left|z_{2}\right|^{2}<1\right\}$ denote the unit ball in $\mathbb{C}^{2}$ and let $S$ denote its boundary, the unit sphere. For $z \in \bar{B}$ and $\delta>0$, the following non isotropic balls are defined, where $\langle z, w\rangle=z_{1} \bar{w}_{1}+z_{2} \bar{w}_{2}$.

$$
B(z, \delta)=\left\{w \in B:|1-\langle z, w\rangle|<\delta^{2}\right\}, \quad S(z, \delta)=\overline{B(z, \delta)} \cap S .
$$

A finite positive Borel measure $\mu$ on $B$ is called a Carleson measure if there exists a constant $C$ for which

$$
\mu(B(\xi, \delta)) \leqslant C \sigma(S(\xi, \delta)), \quad \xi \in S, \quad \delta>0 .
$$

Here $\sigma$ denotes normalized surface area measure on $S$. The following theorem was obtained by Hörmander [6] as a special case of more general variants for strictly pseudoconvex domains in $\mathbb{C}^{n}$. Recently Cima and Wogen [3] derived it from a Carleson measure theorem for Bergman spaces of the ball. A different direct approach to the Bergman context, and related settings, is given in Leucking [7].

THEOREM. Let $\mu$ be a finite Borel measure on $B$. In order that there exist a constant $C$ such that

$$
\int_{B}|f(z)|^{2} d \mu(z) \leqslant C \int_{S}|f(\xi)|^{2} d \sigma(\xi)
$$

for all $f$ in $H^{2}(\sigma)$, it is necessary and sufficient that $\mu$ be a Carleson measure.

We give a natural proof of this theorem and point out connections with Fejér-Riesz type theorems and Hankel operators.

The proof is natural in the sense that it is modelled on Stein's simple proof (see [12]) of Carleson's original theorem [1]. The nontangential maximal function is replaced by the Koranyi maximal function with respect to the approach regions

$$
D(\eta)=\left\{z \in \mathbb{C}^{2}:|1-\langle z, \eta\rangle|<1-|z|^{2}\right\}, \quad \eta \in S .
$$

Note that these regions admit tangential approach. Nevertheless the associated maximal function,

$$
(M f)(\xi)=\sup \{|f(w)|: w \in D(\xi)\}, \quad \xi \in S,
$$

is bounded as an operator from $H^{2}(\sigma)$ to $L^{2}(\sigma)$. (See Rudin [11, Chapter 5 and Theorem 5.4.10].) To transplant the argument we need the following covering lemma.

Lemma. Let $g$ be a continuous function on $B$ and let $a>0$. Then either $|g(w)|<a$ in $B \backslash \frac{1}{2} B$ or there exist points $w_{1}, w_{2}, \ldots$ in $B \backslash \frac{1}{2} B$, possibly finite in number, such that

(i) $\left|g\left(w_{i}\right)\right| \geqslant a, i=1,2, \ldots$,

(ii) $\{w:|g(w)| \geqslant a\} \cap B \backslash \frac{1}{2} B$ is contained in the union of the balls $B\left(w_{i}, 2\left(1-\left|w_{i}\right|^{2}\right)^{1 / 2}\right)$,

Glasgow Math. J. 26 (1985) 13-17. 
$i=1,2, \ldots$,

(iii) the balls $S\left(w_{i},\left(1-\left|w_{i}\right|^{2}\right)^{1 / 2}\right), i=1,2, \ldots$ are disjoint.

Proof. The function $d(z, w)=|1-\langle z, w\rangle|^{1 / 2}$ on $\bar{B} \times \bar{B}$ satisfies the triangle inequality $[11, p .66]$. From this it follows that if $z \notin B\left(w, 2\left(1-|w|^{2}\right)^{1 / 2}\right)$ and $|z| \leqslant|w|$ then $S\left(w,\left(1-|w|^{2}\right)^{1 / 2}\right)$ and $S\left(z,\left(1-|z|^{2}\right)^{1 / 2}\right)$ are disjoint. Indeed, if $w^{1}$ is in the intersection then $d(z, w) \leqslant d\left(w, w^{1}\right)+d\left(w^{1}, z\right)<\left(1-|w|^{2}\right)^{1 / 2}+\left(1-|z|^{2}\right)^{1 / 2}<2\left(1-|w|^{2}\right)^{1 / 2}$, a contradiction. Suppose that $w_{1}, w_{2}, \ldots, w_{n}$ have been chosen so that for $i \neq j, w_{i} \notin B\left(w_{j}, 2\left(1-\left|w_{j}\right|^{2}\right)^{1 / 2}\right)=$ $B_{i}$, say. Pick $w_{n+1}$ in $B \backslash \frac{1}{2} B$ with $w_{n+1} \notin B_{i}, j=1, \ldots, n$, so that $\left|g\left(w_{n+1}\right)\right| \geqslant a$ and $\left|w_{n+1}\right|$ is minimum. If this is impossible then (ii) holds with a finite union. If such a choice is always possible then (ii) will automatically hold for the sequence $w_{1}, w_{2}, \ldots$ so defined. Also (iii) follows from our opening observations.

Proof of the theorem. Throughout the paper various constants which depend only on $\mu$ will be denoted by the universal constant $C$.

We first show sufficiency, which is the harder direction. It can be assumed, without loss, that $\mu$ is supported on $B \backslash \frac{1}{2} B$. (Consider the Cauchy integral formula for the ball $[11, p .38]$ to reduce to this case.) The basic idea is to obtain a distribution function inequality ((7) below) which shows that for $f$ in $H^{2}(\sigma)$

$$
\int_{B}|f|^{2} d \mu \leqslant C \int_{S}|M f|^{2} d \sigma .
$$

The boundedness of the Koranyi maximal function than gives (1). Fix $f$ in $H^{2}$ and $a>0$ and use the covering lemma to obtain $w_{1}, w_{2}, \ldots$ in $B \backslash \frac{1}{2} B$ having the properties (i)-(iii) for $g=|f|$. Observe that

$$
\left\{\eta \in S: w_{i} \in D(\eta)\right\}=S\left(w_{i},\left(1-\left|w_{i}\right|^{2}\right)^{1 / 2}\right),
$$

so that $(M f)(\eta) \geqslant a$ throughout $S\left(w_{i},\left(1-\left|w_{i}\right|^{2}\right)^{1 / 2}\right)$. Thus

and

$$
\mu(\{|f| \geqslant a\}) \leqslant \sum \mu\left(B\left(w_{i}, 2\left(1-\left|w_{i}\right|^{2}\right)^{1 / 2}\right)\right.
$$

$$
\left.\sum \sigma\left(S, w_{i},\left(1-\left|w_{i}\right|^{2}\right)^{1 / 2}\right)\right) \leqslant \sigma(\{M f \geqslant a\}) .
$$

To make the link between (3) and (4) we need to use the Carleson condition and a little geometry. Using the triangle inequality we have

$$
B\left(w, 2\left(1-|w|^{2}\right)^{1 / 2}\right) \subset B(w /|w|, r)
$$

as long as $d(w, w /|w|)+2\left(1-|w|^{2}\right)^{1 / 2}<r$. So this holds for $r=(1+2 \sqrt{2})(1-|w|)^{1 / 2}$. But there is an absolute constant $C_{1}$ such that

$$
\sigma\left(S\left(w /|w|,(1+2 \sqrt{2})(1-|w|)^{1 / 2}\right)\right) \leqslant C_{1} \sigma\left(S\left(w,\left(1-|w|^{2}\right)^{1 / 2}\right)\right)
$$

Combining (5) and (6) with the Carleson condition gives, for all $i$,

$$
\mu\left(B\left(w_{i}, 2\left(1-\left|w_{i}\right|^{2}\right)^{1 / 2}\right)\right) \leqslant C \sigma\left(S\left(w_{i},\left(1-\left|w_{i}\right|^{2}\right)^{1 / 2}\right)\right),
$$


and so, from (3) and (4),

$$
\mu(\{f \mid \geqslant a\}) \leqslant C \sigma(\{M f \geqslant a\}) .
$$

Now (2) follows, completing this part of the proof.

The necessity of the Carleson condition can be shown with natural estimates involving the test functions $\left(1-|\lambda|^{2}\right)(1-\langle z, \lambda\rangle)^{-2}$, for $\lambda$ in $B$. Details are left to the reader.

A Fejér-Riesz inequality. For functions $f$ in the Hardy space of the circle we have the Fejér-Riesz inequality [5, p. 46]

$$
\int_{-1}^{1}|f(x)|^{2} d x \leqslant \pi\|f\|_{2}^{2}
$$

To obtain an analogue for $H^{2}(\sigma)$ it is natural to replace the interval $(-1,1)$ by the unit disc $U=B \cap \mathbb{R}^{2}$ and to replace Lebesgue measure by some natural Carleson measure supported on $U\left(\mathbb{R}^{2}=\left\{\left(z_{1}, z_{2}\right): z_{1}, z_{2}\right.\right.$ are real $\left.\}\right)$. For such measures the Carleson condition simplifies. If $I$ is a subarc of the boundary of $U$ then let $U(I)$ denote the region in $U$ enclosed by $I$ and the chord determined by $I$. Then a positive Borel measure $\nu$, supported by $U$, is a Carleson measure if and only if

$$
\nu(U(I)) \leqslant C|I|^{4}
$$

for all subarcs $I$, where $|I|$ denotes the arclength of $I$. To see this note first that for $\xi \in \partial U$, $\delta>0$, we have $B(\xi, \delta) \cap U=U\left(I_{\xi, \delta}\right)$ where $I_{\xi, \delta}$ is the arc centered at $\xi$ whose chord has midpoint $\left(1-\delta^{2}\right) \xi$. (Sketch the goemetry for $\xi=(1,0)$ and this becomes clear.) Now $\left|I_{\xi . \delta}\right| \delta^{-1} \rightarrow 2 \sqrt{2}$ as $\delta \rightarrow 0$. Also $G(S(\xi, \delta)) \delta^{-4}$ converges to a nonzero limit as $\delta \rightarrow 0[\mathbf{1 1}, \mathrm{p}$. 67 , with different notation]. Consequently condition (8) is equivalent to

$$
\nu(B(\xi, \delta)) \leqslant C \sigma(S(\xi, \delta))
$$

for points $\xi \in \partial U$, and this is equivalent to $\nu$ being a Carleson measure.

COROLlaRY. Let $d U$ denote area measure on the disc $U=B \cap \mathbb{R}^{2}$. Then there is a constant $C$ such that for all $f$ in $H^{2}(\sigma)$.

$$
\int_{U}|f(z)|^{2}\left(1-|z|^{2}\right)^{1 / 2} d U \leqslant C\|f\|_{2}
$$

To see this just check that the measure $d \nu=\left(1-|z|^{2}\right)^{\alpha} d U$ satisfies (8) if and only if $\alpha \geqslant \frac{1}{2}$. This approach gives no insight into the best possible constant $C$ for which (9) is valid. What is this constant?

REMARK. Shields has obtained a version of the Fejér-Riesz inequality for the Dirichlet space [13].

Hankel operators. The Fejér-Riesz inequality is closely allied to the boundedness of the Hilbert matrix $(i+j+1)^{-1}$ as an operator on the classical Hardy space $H^{2}$. This operator plays a distinguished role in the class of Hankel operators on the circle (see [2], [9] for example), and so it is natural to seek analogues on $H^{2}(\sigma)$. 
It is convenient to introduce Hankel operators on $H^{2}(\sigma)$ via Hankel forms on the ring $\mathbb{C}\left[z_{1}, z_{2}\right]$ of complex polynomials in $z=\left(z_{1}, z_{2}\right)$. We define a Hankel form $[p, q]$ as a sesquilinear form such that $[p, q]=\left[p q^{+}, 1\right]$ for all polynomials $p, q$, where $q^{+}(z)=\overline{q(\bar{z})}$ and $\bar{z}=\left(\bar{z}_{1}, \bar{z}_{2}\right)$. Such a form is determined by the coefficient multisequence, $a_{\alpha}=\left[z^{\alpha}, 1\right]$, where $z^{\alpha}$ is the monomial $z^{\alpha_{1}} z^{\alpha_{2}}$. (The finite rank Hankel forms are characterized in [10].) We are interested in Hankel forms which are bounded with respect to $H^{2}(\sigma)$. Indeed it is an immediate consequence of Hörmander's theorem that if $\mu$ is a Carleson measure on $B$ then $[p, q]=\int p q^{+} d \mu$ defines a bounded Hankel form. The most general way to generate a bounded Hankel form is by means of a symbol function $\varphi$ in $L^{\infty}(S)$. In fact the CoifmanRochberg-Weiss weak factorisation of $H^{1}(\sigma)$ functions [4, Theorem III] leads to an analogue of Nehari's theorem for Hankel matrices. Namely, $[\cdot, \cdot]$ is a bounded Hankel form if and only if there exists a function $\varphi$ in $L^{\infty}(\sigma)$ such that

$$
[p, q]=\int_{S} p q^{+} \varphi d \sigma
$$

To identity the operator on $H^{2}(\sigma)$ which implements the form, let $J$ be the unitary operator on $L^{2}(\sigma)$ such that $(J f)(\xi)=f(\bar{\xi})$, and let $P$ be the Hardy space projection. Then $[p, q]=(\varphi p, J q)=\left(J_{\varphi} p, q\right)=\left(S_{\varphi} p, q\right)$ where $S_{\varphi}=P J M_{\varphi}$ as an operator on $H^{2}(\sigma)$, and $M_{\varphi}$ denotes multiplication by $\varphi$.

The following proposition gives an alternative proof of the boundedness of the measures $\left(1-|z|^{2}\right)^{\alpha} d U$, for $\alpha>\frac{1}{2}$. (Observe that $\int|f|^{2} d \mu=\int f f^{+} d \mu$ when $\mu$ is supported on $U$.) Since the associated symbol functions are continuous it follows that the associated Hankel operators are compact operators (see [4], or use the elementary [10, Lemma 5] and some uniform approximation). However, the operator, $H$ say, associated with the measure $\left(1-|z|^{2}\right)^{1 / 2} d U$, is not compact. $H$ seems to be reminiscent of a Hilbert matrix and it is natural to enquire,

(a) is there a natural symbol function for $H$,

(b) what is $\|H\|$ (cf. inequality (9)),

(c) what is the spectrum of $H$ (cf. [8], [9])?

Proposmion. Let $U$ be the unit disc $B \cap \mathbb{R}^{2}$ with area measure $d U$ and let $\alpha>\frac{1}{2}$. Then for $h$ in $H^{2}(\sigma)$

$$
\int_{U} h(w)\left(1-|w|^{2}\right)^{\alpha} d U=\int_{S} f(\xi) \varphi(\xi) d \sigma(\xi)
$$

where $\varphi$ is the continuous coanalytic function on the sphere $S$ such that for $\xi \notin S \cap \mathbb{P}^{2}$

$$
\varphi(\xi)=2 \pi \int_{0}^{1} \frac{\left(1-\rho^{2}\right)^{\alpha} \rho}{\left(1-\rho^{2}\left(\bar{\xi}_{1}^{2}+\bar{\xi}_{2}^{2}\right)\right)^{3 / 2}} d \rho .
$$

Proof. Fix $f$ in $H^{1}(\sigma)$ and let $C_{w}(\xi)=(1-\langle w, \xi\rangle)^{-2}$ be the Cauchy kernel so that $f(w)=\left(f, \bar{C}_{w}\right)$ for $w$ in $B$. Parametrise points $w$ in $U$ by $w=(\rho \cos \theta, \rho \sin \theta)$. Then, for 
$0<r<1$,

where

$$
\begin{aligned}
\int_{r U} f(w)\left(1-|w|^{2}\right)^{\alpha} d U & =\int_{r U} \int_{S} f(\xi) C_{w}(\xi) d \sigma(\xi)\left(1-\left|w^{2}\right|\right)^{\alpha} d U(w) \\
& =\int_{S} f(\xi) \varphi_{r}(\xi) d \sigma(\xi)
\end{aligned}
$$

$$
\varphi_{r}(\xi)=\int_{r U}\left(1-\bar{\xi}_{1} \rho \cos \theta-\bar{\xi}_{2} \rho \sin \theta\right)^{-2}\left(1-\rho^{2}\right)^{\alpha} \rho d \rho d \theta
$$

Using contour integration for the $\theta$ integral $[11$, p. 244] this becomes

$$
2 \pi \int_{0}^{r} \frac{\left(1-\rho^{2}\right)^{\alpha} \rho}{\left(1-\rho^{2}\left(\bar{\xi}_{1}+\bar{\xi}_{2}^{2}\right)\right)^{3 / 2}} d \rho .
$$

Let $r \rightarrow 1$ and (11) and (12) follow. For $\alpha>\frac{1}{2}$ the function $\varphi$ is continuous when defined to vanish on $S \cap \mathbb{R}^{2}$.

\section{REFERENCES}

1. L. Carleson, Interpolation by bounded analytic functions and the corona problem, Ann. of Math. (2) 76 (1962), 547-599.

2. M.-D. Choi, Tricks or treats with the Hilbert matrix, Amer. Math. Monthly, 90 (1983), 301-312.

3. J. A. Cima and W. R. Wogen, A Carleson measure theorem for the Bergman space on the ball, J. Operator Theory 7 (1982), 157-165.

4. R. R. Coifman, R. Rochberg and G. Weiss, Factorisation theorems for Hardy spaces in several variables, Ann. of Math. (2) 103 (1976), 611-635.

5. P. L. Duren, Theory of $H^{D}$ spaces (Academic Press, 1970). 65-78.

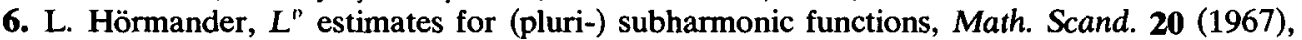

7. D. Leucking, A technique for characterising Carleson measures on Bergman spaces, Proc. Amer. Math. Soc. 87 (1983), 656-660.

8. W. Magnus, On the spectrum of Hilbert's matrix, Amer. J. Math. 72 (1950), 699-704.

9. S. C. Power, Hankel operators on Hilbert space, Research Notes in Mathematics, No. 64 (Pitman, 1982).

10. S. C. Power, Finite rank multivariable Hankel forms, Linear Algebra Appl. 48 (1982), 237-244.

11. W. Rudin, Function theory in the unit ball of $C^{n}$ (Springer-Verlag, 1980).

12. D. Sarason, Function theory on the unit circle, Lecture notes for a conference at Virginia Polytechnic and State University, Blacksburg, Virginia, June 1978.

13. A. L. Shields, An analogue of the Fejér-Riesz theorem for the Dirichlet space, in W. Beckner et al., eds. Conference on harmonic analysis in honour of Antoni Zygmund (Wadsworth, 1983), Vol. II, 810.

Department of Mathematics

Michigan State UnIVERSTTY

East Lansing, Michigan 48824

U.S.A.
Department of Mathematics

UNIVERSTTY OF LANCASTER

BaIlRigg, Lancaster LA1 4YW

ENGLAND 